\title{
Editorial
}

\section{Transcriptional and Post-Transcriptional Regulation in Hormone-Dependent Cancer}

The special issue provides a compilation of five review articles and five research articles on ETS transcriptional control, microRNA regulation of mRNA degradation and translation, post-transcriptional control of pre-RNA processing, protein stability and degradation. Thus, this issue highlights the areas that represent the main control mechanisms of gene expression from primary transcription to protein expression.

ETS factors are involved in cancer progression through transcriptional regulation of factors mediating cell cycle, cell growth, apoptosis, cell adhesion and migration. Dr. Dennis Watson and colleagues begin this issue with a review of ETS transcription factors in prostate and breast cancer. In addition to tabulation of the available data on ETS factor expression in tumor versus normal tissue, their review highlights the functional significance of some of the observed changes. It is becoming widely accepted that more than a single ETS factor is able to regulate any one gene; therefore, this review also puts forth the model that the precise balance between cancer promotion and inhibition by multiple ETS factors may differentially regulate specific target genes, and, thus, control its progression.

In the second review, Dr. David Turner presents a focused overview of published observations that support the hypothesis that multiple ETS factors function together with the androgen receptor to regulate pathways that control progression from hormone sensitive to hormone refractory prostate cancer.

For several years, it has become well established that the tumor microenvironment provides a critical component in cancer progression. Thus, it has become important to focus not only on the tumor epithelial cells but also on non-epithelial stromal cells, including fibroblasts, endothelial cells and infiltrating cells. Dr. Michael Ostrowski's review describes some of the critical regulatory roles defined for specific ETS factors in stromal cells during both development and cancer. This discussion supports the notion that ETS signaling is important not only in tumor cells, but also in the surrounding cells comprising the tumor microenvironment.

Dr. Victoria Findlay presents an overview of microRNAs, a group of small non-coding RNAs that are involved in the negative post-transcriptional regulation of gene expression, and their role in processes related to breast cancer progression. While much initial effort had been placed upon the power of microRNA profiling to distinguish specific cancer types and subgroups, this review discusses current and future efforts directed towards understanding the role of specific microRNAs and their target genes in normal breast and cancer development and progression.

Alternative splicing of pre-mRNA is a post-transcriptional event that generates multiple mRNAs from a primary transcript. As described for microRNAs, the article by Dr. Patricia Watson reviews data supporting the notion that mRNA splice variant expression patterns have the power to sub-classify tumor tissues and specific variants may also be better predictors of disease 
outcome. In addition to reviewing published alternative splicing products, Dr. Watson highlights the role of specific splice variants, and/or the proteins they encode, in prostate and breast cancer.

These reviews are followed by a series of original research articles. The first two provide new insights into the functional significance of ESE-1 (ESX) over-expression in breast cancer progression. Data from the laboratory of Dr. Arthur GutierrezHartmann demonstrates that ESE-1 expression is required for transformed phenotypes of breast cancer cells. Drs. Richard Neve, Christopher Benz and colleagues present data supporting an ERBB2-ESE-1 regulatory axis and its contribution to breast cancer invasiveness and progression.

Drs. Albert Dobi, Shiv Srivastava and colleagues were among the first to demonstrate that the ETS transcription factor, ERG, was over-expressed in the majority of prostate cancer specimens. In their article, they provide data demonstrating that $E R G$ is an androgen receptor regulated gene and tumors with lower $E R G$ expression represent a subset with attenuated AR signaling.

The article from Dr. Charles Bieberich examines the role of post-translational modification on the stability of the ETS factor, PDEF. Data is provided to show that PDEF is a substrate for the protein kinase CK2, define the CK2 phosphoacceptor sites on PDEF, and show that inhibition of CK2 is associated with proteosome-mediated PDEF degradation.

The addition of ubiquitin or SUMO can control protein stability. The article by Drs. Angelika Burger, Arun Seth and colleagues presents data supporting the model that BCA2 (Breast Cancer Associated gene 2), a novel RING-finger ubiquitin E3 ligase, may function as an E3 ligase in the sumoylation of transcription factors. This manuscript also shows that BCA2 is estrogen regulated and co-expressed with the estrogen receptor (ER) in most ER-positive invasive ductal breast cancer, and discusses the potential involvement of the $\mathrm{BCA} 2$ in hormone responsive breast cancer.

Dennis K. Watson

(Guest Editor)

Hollings Cancer Center Medical University of South Carolina Charleston, SC, 29425

USA

Tel: 843-792-3962

E-mail: watsondk@musc.edu

(C) Dennis K. Watson; Licensee Bentham Open.

This is an open access article licensed under the terms of the Creative Commons Attribution Non-Commercial License (http://creativecommons.org/licenses/by$\mathrm{nc} / 3.0 /$ ), which permits unrestricted, non-commercial use, distribution and reproduction in any medium, provided the work is properly cited. 